\title{
Mesures des propriétés cycliques des sols limoneux ou argileux au laboratoire
}

\author{
Jean-François Serratrice ${ }^{\star}$ \\ Cerema, direction territoriale Méditerranée, 30, rue Albert-Einstein, CS 70499, 13593 Aix-en-Provence, France
}

\begin{abstract}
Résumé - Une méthode d'exploitation des essais triaxiaux cycliques est proposée pour identifier les propriétés dynamiques des sols fins limoneux ou argileux au laboratoire. L'essai consiste à appliquer plusieurs séquences successives de cycles en condition non drainée dans une gamme de déformations axiales comprises entre $10^{-4}$ et $10^{-2}$. Une approximation des courbes expérimentales permet d'effectuer l'identification précise du module d'Young sécant et de l'amortissement. L'exploitation d'un essai donne une illustration de la méthode.
\end{abstract}

Mots clés : argile / laboratoire / triaxial / module / amortissement

\begin{abstract}
Cyclic triaxial measurements on silty and clayey soils in the laboratory. An operating method for cyclic triaxial testing is proposed to identify the dynamic properties of silty or clayey fine soils in the laboratory. The test consists in applying several successive sequences of undrained cycles in a range of axial deformations between $10^{-4}$ and $10^{-2}$. An approximation of the experimental records allows the precise identification of secant Young modulus and damping. An operating test gives an illustration of the method.
\end{abstract}

Keywords: clay / laboratory / triaxial / modulus / damping

\section{Introduction}

La connaissance du comportement cyclique des sols s'impose pour comprendre la réponse des sites quand ils sont frappés par un séisme. En très petites déformations, la raideur du sol est représentée par le module de cisaillement $G_{\max }$ qui est en lien direct avec la vitesse des ondes de cisaillement $V_{\mathrm{s}}$. Dans la gamme des déformations faibles à moyennes, le comportement des sols est non linéaire et hystérétique. La raideur du sol est représentée par le module de cisaillement sécant $G$. Il est d'usage de l'exprimer aussi par le rapport $G / G_{\max }$. La dissipation d'énergie par le sol au cours des cycles est représentée par le coefficient d'amortissement $D$. L'existence d'un amortissement minimal $D_{\min }$ en très petite déformation est admise généralement. Comme beaucoup de propriétés mécaniques des sols, $G$ et $D$ dépendent à la fois de la contrainte effective de confinement $\sigma_{\mathrm{m}}$ et du niveau de déformation de cisaillement $\gamma$.

En pratique, le recueil des propriétés dynamiques des terrains s'effectue par la mesure directe des vitesses $V_{\mathrm{s}}$ in situ, ou une estimation indirecte de celles-ci, puis au laboratoire, où sont mesurées ou estimées les variations de $G$ et $D$ avec $\sigma^{\prime}{ }_{\mathrm{m}}$ et $\gamma$. En reproduisant des sollicitations cycliques appropriées et contrôlées, les essais de laboratoire permettent d'identifier les paramètres représentatifs du comportement non linéaire et

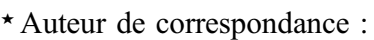

jean-francois.serratrice@cerema.fr
}

hystérétique des sols (colonne résonnante, cisaillement simple, triaxial). Dans cette démarche, qui s'impose pour les projets importants, il est impératif de disposer d'échantillons carottés de bonne qualité et représentatifs des sols.

Avec le temps, beaucoup de données expérimentales ont été recueillies au laboratoire en matière de propriétés dynamiques d'une large variété de sols dans le monde. Deux appareillages complémentaires sont d'usage courant, la colonne résonnante pour les petites déformations $\left(10^{-6}\right.$ à $\left.10^{-4}\right)$ et le triaxial pour les déformations faibles à moyennes $\left(10^{-4}\right.$ à $\left.10^{-2}\right)$. La norme américaine ASTM D 3999-91 «Standard Test Method for the determination of the modulus and damping properties of soils using cyclic triaxial apparatus » (ASTM, 1996) constitue une procédure de référence concernant l'essai triaxial. Les compilations des nombreuses données expérimentales disponibles dans la littérature ont abouti à diverses expressions empiriques donnant le module de cisaillement $G$ et de l'amortissement $D$ en fonction des principaux facteurs qui gouvernent ces paramètres.

Malgré toutes ces connaissances accumulées, le raffinement des pratiques d'essai et les avancées technologiques en matière de matériels, la mesure des propriétés dynamiques des sols fins dans leur état naturel au laboratoire reste problématique, en ce qui concerne l'amortissement notamment, qui est sensible aux conditions d'essai. Dans ce sens, une méthode d'exploitation des courbes d'essai triaxial cyclique est proposée ici. Elle s'appuie sur une approximation en séries de Fourier des courbes expérimentales en vue d'en éliminer le bruit afin d'accéder à l'identification précise du module 
d'Young sécant et de l'amortissement dans la gamme des amplitudes faibles à moyennes des déformations cycliques. L'exploitation d'un essai triaxial cyclique donne une illustration de la méthode. Le sol testé est un limon dont les propriétés physiques et mécaniques sont décrites, avant de passer à l'identification de ses caractéristiques dynamiques.

\section{Propriétés dynamiques des sols}

\subsection{Aspects généraux}

Ce paragraphe donne un aperçu des propriétés dynamiques des sols du point de vue des essais de laboratoire en référence à Seed et Idriss (1970), Hardin et Drnevich (1972b), Iwasaki et Tatsuoka (1977), Iwasaki et al. (1978), Zen et al. (1978), Kokusho (1980), Kokusho et al. (1982), Seed et al. (1986), Sun et al. (1988), Vucetic et Dobry (1991), Ishibashi et Zhang (1993), Rollins et al. (1998), Vucetic et al. (1998), Darendeli (2001), Stokoe et al. (1999), Stokoe et al. (2004), Zhang et al. (2005), Bui et al. (2007), Guerreiro et al. (2012), Liao et al. (2013), Vardanega et Bolton (2013), El Mohtar et al. (2013), parmi d'autres.

Le module $G$ et le rapport $G / G_{\max }$ dépendent des nombreux paramètres caractéristiques du comportement mécanique des sols testés dans leur état naturel. Ces paramètres dépendent de la nature des sols, en lien notamment avec l'indice de plasticité $I_{\mathrm{p}}$ pour les sols argileux, ou la granulométrie et la forme des grains pour les sables. L'âge du dépôt intervient. Les paramètres dépendent aussi de l'état du sol en matière de compacité (indice des vides, indice de consistance pour les argiles, indice de densité pour les sables), d'histoire passée (surconsolidation) et de saturation. Ils expriment l'anisotropie plus ou moins marquée du sol. Ils dépendent de l'état initial (contrainte moyenne effective $\sigma_{\mathrm{m}}^{\prime}$ et rapport des contraintes initiales), de l'amplitude de la déformation et de la vitesse de chargement (fréquence), puis du nombre de cycles. Généralement, dans les sols argileux, la dégradation du rapport $G / G_{\max }$ avec $\gamma$ est moins rapide quand $\sigma_{\mathrm{m}}^{\prime}$ et $I_{\mathrm{p}}$ augmentent. Les sols peu plastiques sont plus dépendants de $\sigma_{\mathrm{m}}^{\prime}$ que les sols plastiques.

L'amortissement $D$ dépend aussi des paramètres évoqués ci-dessus (nature du sol, état du sol, conditions initiales et chargements). Toutefois, considéré comme amortissement hystérétique, il est supposé indépendant de la vitesse de chargement (fréquence) par opposition à un amortissement visqueux. Ainsi, $D$ augmente avec $\gamma$ dans la gamme des petites aux moyennes déformations. La connaissance de $D_{\min }$ à très petite déformation et de $D$ à grande déformation est moins précise. La mesure s'avère difficile du fait de l'influence de la machine d'essai et des conditions d'essai (fréquence, nombre de cycles, condition de contact des éprouvettes) ou de l'accumulation des déformations irréversibles.

Parmi tous les facteurs évoqués ci-dessus et largement étudiés, il n'est pas fait mention des effets de la pression interstitielle. Pourtant, les essais triaxiaux cycliques sont réalisés en condition non drainée pour demeurer en accord avec les fréquences sismiques. Dans la gamme des petites aux moyennes déformations et au-delà, l'accumulation de la pression interstitielle pendant les cycles produit la réduction de la contrainte effective $\sigma^{\prime}$. La pression interstitielle agit donc directement sur la mesure des propriétés dynamiques du sol, via l'influence de $\sigma_{\mathrm{m}}^{\prime}$. Elle joue aussi sur l'accumulation des déformations plastiques au cours des cycles. Dans les sables lâches, l'accumulation de la pression interstitielle peut aboutir à l'annulation des contraintes effectives et à la liquéfaction du sol accompagnée d'une dégradation quasi totale du module $G$. Ainsi, l'eau interstitielle intervient sur la raideur et l'amortissement du sol en fonction du niveau de sollicitation cyclique.

Dans les argiles, l'effet de cette accumulation est moins tranché. Cet accroissement au cours des cycles ne se fait sentir qu'à partir d'un seuil de l'ordre de $10^{-3}$ de la déformation de cisaillement au plus (Matasovic et Vucetic, 1992). Par la suite la dégradation du module s'opère continûment pendant les cycles sous l'effet d'une accumulation de la pression interstitielle, qui s'effectue dans une progression linéaire avec le logarithme du nombre de cycles le plus souvent (Matasovic et Vucetic, 1995). De fait, comme dans les sables, l'identification des propriétés dynamiques du sol n'est pas effectuée dans un état de contraintes effectives constantes.

\subsection{Description empirique}

Après avoir compilé de nombreux résultats expérimentaux portant sur différents types de sols, différents appareillages et différents types d'essais cycliques, Vucetic et Dobry (1991) ont établi un faisceau de courbes qui permet de décrire la dégradation du module de cisaillement $G$ et de l'amortissement $D$ en fonction de la déformation de cisaillement et de l'indice de plasticité. Puis, Ishibashi et Zhang (1993) ont développé une expression analytique donnant $G$ et $D$ en fonction du module maximal $G_{\max }$, de l'amplitude de la déformation cyclique de cisaillement, de la contrainte effective de confinement et de l'indice de plasticité $I_{\mathrm{p}}$ de l'argile. Une approche possible pour caractériser l'amortissement consiste à relier celui-ci au rapport $G / G_{\max }$. Un avantage de cette approche tient à sa meilleure robustesse, $\operatorname{car} D$ est plus difficile à mesurer avec précision que le rapport $G / G_{\max }$. Hardin et Drnevich (1972a) ont suggéré de relier $D$ proportionnellement à $1-G / G_{\max }$. Ishibashi et Zhang (1993) ont proposé une relation polynomiale de $G / G_{\max }$.

De nombreuses formulations ont été établies par la suite. Vardanega et Bolton (2013) présentent une expression semiempirique de $G_{\max }$ fonction de l'indice des vides et de la contrainte effective de confinement $\sigma_{\mathrm{m}}^{\prime}$. L'effet de la vitesse de chargement est pris en compte, en se fondant à la fois sur des essais de laboratoire monotones et cycliques, pour des applications aux problèmes de chargements statiques (fondations) ou dynamiques (séismes).

\section{Description des courbes triaxiales cycliques}

Une méthode de description des courbes triaxiales cycliques est proposée ici en vue de l'identification des propriétés dynamiques du sol dans la gamme des déformations petites à moyennes.

\subsection{Procédure d'essai}

Les essais triaxiaux cycliques destinés à la mesure des propriétés dynamiques des sols se déroulent en plusieurs phases. Pour les aspects conventionnels, la réalisation de l'essai se réfère à la norme de l'essai triaxial à chargement monotone « Essais à l'appareil triaxial de révolution. Appareillage. 
Préparation des éprouvettes. Essais UU non consolidé non drainé. Essai $\mathrm{CU}+u$ consolidé non drainé avec mesure de la pression interstitielle. Essai CD consolidé drainé » (AFNOR, 1994a,b). Un exemple d'essai triaxial à chargement monotone réalisé sur le limon sera décrit plus bas. Pour les aspects cycliques il est fait référence à la norme américaine ASTM D 3999-91 citée plus haut. Quelques éléments pratiques sont indiqués ci-dessous.

Après découpage, l'éprouvette est placée dans la cellule triaxiale. Celle-ci est munie d'un dispositif d'extension. Il est recommandé d'utiliser un dispositif de mesure locale de la déformation fixé sur l'éprouvette. La saturation du sol est parachevée par l'application de paliers de contre-pression. L'éprouvette est consolidée sous une pression effective isotrope $p_{\mathrm{c}}$ définie en fonction des conditions de site (la profondeur dans le massif ou le chargement sous l'ouvrage).

La sollicitation cyclique non drainée est appliquée ensuite. Elle est composée par plusieurs séquences de cycles successives pilotées en déplacement (essai étagé en mode DF, contrôle en déplacement et en fréquence). Le déplacement axial sinusoïdal alterné, qui est imposé, est défini par une amplitude $\Delta h$ et une fréquence $f$. La sollicitation cyclique est maintenue constante jusqu'à atteindre un nombre fixé de cycles. À cette amplitude cyclique correspond l'amplitude de la déformation axiale $\Delta \varepsilon_{\mathrm{a}}$ (notée aussi $\Delta \varepsilon_{\mathrm{a}}$ SA pour simple amplitude). Cette amplitude $\Delta \varepsilon_{\mathrm{a}}$ est augmentée d'une séquence de cycles à l'autre afin de balayer la gamme des déformations axiales comprises entre $10^{-4}$ et $10^{-2}$ (de $0,01 \%$ à $1 \%$ ). La reconsolidation préalable de l'éprouvette sous la pression effective isotrope $p_{\mathrm{c}}$ est effectuée avant de commencer une nouvelle séquence si la pression interstitielle accumulée devient trop grande pendant la séquence précédente.

Pendant les séquences de cisaillement cyclique, le déplacement axial $\Delta h$ (mesures globale et locale), l'effort axial $F$ et la pression interstitielle $u$ sont enregistrés en continu. L'échantillonnage des mesures est réalisé à pas de temps constant à raison de 50 ou 100 points par cycle. Ces mesures sont converties en déformation axiale $\varepsilon_{\mathrm{a}}$, en pression moyenne effective $p=\left(\sigma^{\prime}{ }_{\mathrm{a}}+2 \sigma{ }_{\mathrm{r}}\right) / 3$ et en déviateur $q=\sigma^{{ }_{\mathrm{a}}}$ $-\sigma_{\mathrm{r}}$, où $\sigma_{\mathrm{a}}$ et $\sigma_{\mathrm{r}}$ sont les contraintes effectives axiale et radiale (la notation $p$ s'adresse bien à la pression moyenne effective en lien avec la notation $p_{\mathrm{T}}$ réservée à la pression moyenne totale et telle que $p=p_{\mathrm{T}}-u$ ). Pendant chaque séquence de cycles, la déformation axiale $\varepsilon_{\mathrm{a}}$ représente la sollicitation d'amplitude $\Delta \varepsilon_{\mathrm{a}} \mathrm{SA}$, à une fréquence donnée et sous une pression de confinement donnée, tandis que le déviateur $q$ constitue la réponse du sol. Les déformations et les contraintes sont comptées positivement en compression. Le calcul de ces variables est effectué à partir des données brutes, en tenant compte de la variation de section de l'éprouvette (forme de déformation en tonneau). Pendant chacune des séquences de cycles, ces variables sont représentées graphiquement en fonction du temps $\left(t, \varepsilon_{\mathrm{a}}, q, u\right)$ et sous la forme de courbe contrainte déformation $\left(\varepsilon_{\mathrm{a}}, q\right),\left(\varepsilon_{\mathrm{a}}, u\right)$ et de chemin des contraintes effectives $(p, q)$.

\subsection{Module d'Young et amortissement}

Dans son principe, l'essai triaxial, pendant lequel la pression de confinement est maintenue constante, permet de mesurer le module d'Young $E$, rapport entre le déviateur $q$ et la déformation axiale $\varepsilon_{\mathrm{a}}$. Un essai non drainé permet de mesurer le module d'Young non drainé $E_{\mathrm{u}}$, dans une plage où la pression interstitielle ne change pas trop. Ce module est directement lié au module de cisaillement $G$. Dans les essais triaxiaux cycliques, le module d'Young non drainé sécant représente la pente moyenne de la boucle contrainte-déformation $\left(\varepsilon_{\mathrm{a}}, q\right)$. D'autres définitions des modules concernent les modules tangents. L'amortissement $D$ traduit la dissipation de l'énergie par le sol pendant les cycles de chargement-déchargement. Fonction de l'aire des boucles d'hystérésis, il se mesure aussi sur les courbes contrainte-déformation $\left(\varepsilon_{\mathrm{a}}, q\right)$. Ces courbes constituent le support pour l'identification des paramètres dynamiques du sol $E_{\mathrm{u}}$ et $D$ pendant les essais triaxiaux cycliques. D'où l'intérêt d'une description précise de ces courbes.

Dans les sols fins limoneux ou argileux la progression du déviateur est continue pendant chacune des séquences de cycles et s'amortit progressivement. Elle est retardée par rapport à la sollicitation qui est imposée en déformation. Toutefois, les boucles contrainte-déformation $\left(\varepsilon_{\mathrm{a}}, q\right)$ se distordent peu à peu quand l'amplitude $\Delta \varepsilon_{\mathrm{a}}$ est augmentée d'une séquence de cycles à l'autre, pour s'éloigner progressivement de la réponse hystérétique. Les boucles $\left(\varepsilon_{\mathrm{a}}, q\right)$ ne sont plus fermées. La norme ASTM D 3999-91 précise les conditions d'exploitation de l'essai triaxial à ce titre. En particulier, l'écart de fermeture $\Delta \varepsilon_{\mathrm{a}}$ de chaque boucle $\left(\varepsilon_{\mathrm{a}}, q\right)$ doit rester inférieur à $0,2 \%$ pour être exploitable. Dans les sables, les amplitudes supérieures à $10^{-3}$ conduisent souvent à des fortes déformations plastiques et une dégrada tion marquée du module $E_{\mathrm{u}}$ (mobilité cyclique avec accumulation continue des déformations irréversibles dans les sables denses, perte totale de raideur par liquéfaction dans les sables lâches).

\subsection{Représentation des courbes en fonction du temps}

L'identification des paramètres dynamiques $E_{\mathrm{u}}$ et $D$ du sol passe par une bonne description des courbes contraintedéformation $\left(\varepsilon_{\mathrm{a}}, q\right)$. Dans ce but, la méthode d'exploitation des essais triaxiaux cycliques proposée ici veut répondre à deux objectifs :

- améliorer l'exploitation des essais en tenant compte de

la forme compliquée des cycles dans la gamme des déformations moyennes ;

- réduire l'effet du bruit des mesures, surtout aux faibles amplitudes.

En effet, il n'existe pas de méthode pour déterminer les propriétés dynamiques à partir de boucles d'hystérésis déformées. Kumar et al. (2015) proposent une technique pour évaluer l'énergie emmagasinée dans les cycles dans ce cas.

Une autre proposition est suggérée ici. Chaque séquence de cycles contrôlée en mode DF fait l'objet d'une exploitation séparée (contrôle en déplacement et en fréquence). Pour chacune de ces séquences, des approximations des courbes enregistrées en fonction du temps $\varepsilon_{\mathrm{a}}(t)$ et $q(t)$ sont calculées au sens des moindres carrés à l'aide de séries de Fourier (approximation de Bessel). 
Ces approximations s'expriment sous la forme :

$$
\begin{aligned}
y(t) & =a_{0} / 2+\sum_{\mathrm{k}=1^{n}} a_{k} \cos (2 \pi k t / T) \\
& +\sum_{\mathrm{k}=1^{n}} b_{k} \sin (2 \pi k t / T),
\end{aligned}
$$

où $a_{\mathrm{k}}$ et $b_{\mathrm{k}}$ sont des amplitudes, $T$ est la durée de la séquence des $N$ cycles et $2 n$ le nombre de mesures. Les coefficients $a_{\mathrm{k}}$ et $b_{\mathrm{k}}$ sont calculés par la méthode des moindres carrés. Les fréquences mineures sont éliminées alors, pour ne conserver que les fréquences dominantes. Cette réduction permet d'effectuer un filtrage des hautes fréquences et d'éliminer ainsi le bruit des mesures des enregistrements $\varepsilon_{\mathrm{a}}(t)$ et $q(t)$. Le calcul est effectué pour tous les cycles de la séquence ou pour un nombre donné de cycles, à définir. Il peut s'agir des dix derniers cycles d'une séquence de cinquante cycles, dans le cas d'une argile par exemple, quand la réponse fait apparaître une stabilisation progressive des cycles (adaptation). Dans les sols fins limoneux ou argileux, ce choix permet de s'éloigner du début de la séquence des cycles où les variations du déviateur et de la pression interstitielle sont souvent les plus fortes, pour ne retenir que les cycles les mieux stabilisés. Mais il peut s'agir des cycles 2 à 5 où des cycles 10 à 20 pour effectuer un choix en lien avec les conditions sismiques du site, en référence à un nombre de cycles équivalent.

\subsection{Représentation des boucles contrainte-déformation}

Pour chacune des séquences de cycles et les cycles sélectionnés, les courbes lissées $\varepsilon_{\mathrm{a}}(t)$ et $q(t)$ sont associées pour former la courbe contrainte-déformation $\left(\varepsilon_{\mathrm{a}}, q\right)$ en vue d'identifier les paramètres dynamiques du sol. Le graphique de la Figure 1 montre deux boucles d'une telle courbe contrainte-déformation $\left(\varepsilon_{\mathrm{a}}, q\right)$. La première boucle représente le cycle $\mathrm{n}^{\mathrm{o}} 1$, l'autre, le cycle $n$. L'origine de la boucle a pour coordonnées $\left(\varepsilon_{\mathrm{a} 0}, q_{0}\right)$. Le cycle $n$ est caractérisé par les doublesamplitudes $\Delta \varepsilon_{\mathrm{a}}$ DA et $\Delta q_{\mathrm{DA}}$. Mais des amplitudes distinctes apparaissent en compression $\Delta \varepsilon_{\mathrm{a}} \mathrm{c}, \Delta q_{\mathrm{c}}$ et en extension $\Delta \varepsilon_{\mathrm{a}}$ e, $\Delta q_{\mathrm{e}}$, en raison de la distorsion du cycle. De ces caractéristiques sont déduits les modules sécants en extension $E_{\mathrm{e}}$ et en compression $E_{\mathrm{c}}$, puis le module global $E_{\mathrm{t}}$ :

$$
\begin{aligned}
& E_{\mathrm{e}}=\Delta q_{\mathrm{e}} / \Delta \varepsilon_{\mathrm{a}} \quad E_{\mathrm{c}}=\Delta q_{\mathrm{c}} / \Delta \varepsilon_{\mathrm{ac}} \\
& E_{t}=\Delta q_{\mathrm{DA}} / \Delta \varepsilon_{\mathrm{a} D A} .
\end{aligned}
$$

Ces modules sécants constituent les pentes des segments indiqués sur le graphique de la Figure 1. L'amortissement $D$ traduit l'énergie dissipée par le sol sous la forme de chaleur pendant les cycles. Il est défini par le rapport entre cette énergie dissipée pendant un cycle (l'aire $A_{\mathrm{c}}$ de la boucle) et l'énergie maximale stockée pendant ce cycle (l'aire du triangle $A_{\mathrm{t}}=\Delta \varepsilon_{\mathrm{a}} \mathrm{c}, \times \Delta q_{\mathrm{c}} / 2$ sous la demi-boucle, d'après les notations de la Fig. 1) :

$$
D=A_{c} / 4 \pi A_{\mathrm{t}} \text {. }
$$

Les aires $A_{\mathrm{c}}$ et $A_{\mathrm{t}}$ sont indiquées sur le graphique de la Figure 1. Sous une sollicitation harmonique $\varepsilon_{\mathrm{a}}(t)$ comme ici et une réponse harmonique $q(t)$, cet amortissement hystérétique représente aussi le déphasage $\phi$ entre les deux signaux et $D=\sin (\phi) / 2$ (hystérésis signifie « en retard ). La courbe

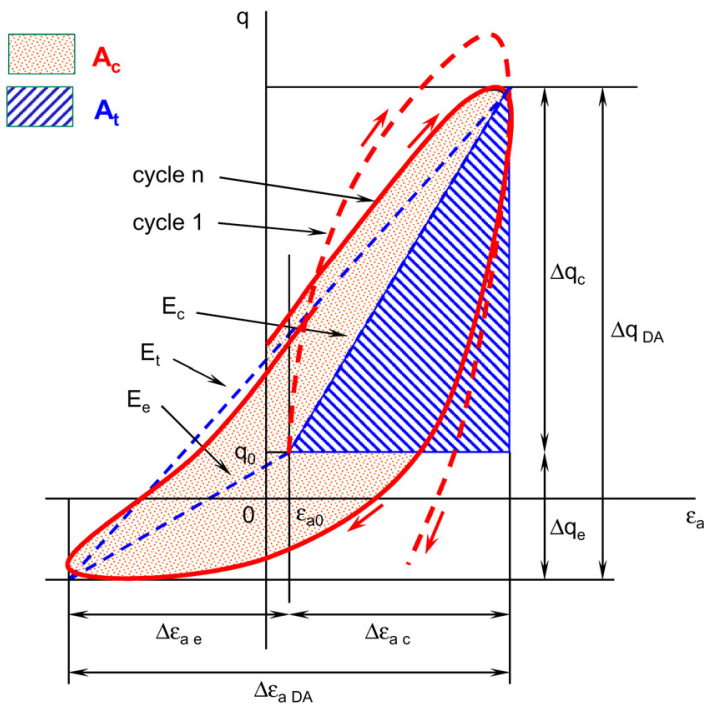

Fig. 1. Paramètres caractéristiques des courbes déformation axiale-déviateur.

Fig. 1. Parameters for deviator stress versus axial strain curves.

contrainte-déformation $\left(\varepsilon_{\mathrm{a}}, q\right)$ est une ellipse dont l'aire ne dépend que de $\phi$. La réponse est indépendante de la vitesse de chargement (fréquence).

La distorsion des boucles $\left(\varepsilon_{\mathrm{a}}, q\right)$ de la Figure 1 montre bien l'approximation que constitue le modèle de comportement hystérétique pour les amplitudes des déformations moyennes à grandes. Les modules $E_{\mathrm{c}}$ et $E_{\mathrm{t}}$ sont différents, les boucles ne sont pas fermées. Aussi, la méthode d'exploitation des essais cycliques proposée ici vise à améliorer l'identification des paramètres dynamiques du sol dans ce cas. En pratique, le lissage des enregistrements $\varepsilon_{\mathrm{a}}(t)$ et $q(t)(1)$ permet de pointer : - les amplitudes cycliques de la déformation axiale et du déviateur en compression et en extension, $\Delta \varepsilon_{\mathrm{a}} \mathrm{c}, \Delta \varepsilon_{\mathrm{a}}$ e, $\Delta q_{\mathrm{c}}$ et $\Delta q_{\mathrm{e}}$, à partir de l'origine $\left(\varepsilon_{\mathrm{a} 0}, q_{0}\right)$ des cycles ;

- les doubles-amplitudes cycliques $\Delta \varepsilon_{\mathrm{a} \text { DA }}=\Delta \varepsilon_{\mathrm{a}} \mathrm{c}-\Delta \varepsilon_{\mathrm{a}}$ e et $\Delta q_{\mathrm{DA}}=\Delta q_{\mathrm{c}}-\Delta q_{\mathrm{e}}$;

- puis de calculer l'aire $A_{\mathrm{c}}$ des boucles $\left(\varepsilon_{\mathrm{a}}, q\right)$ pendant chacun des cycles ;

- l'aire du triangle situé du côté des compressions $A_{\mathrm{t}}=\Delta \varepsilon_{\mathrm{a}} \mathrm{c}$ $\times \Delta q_{\mathrm{c}} / 2$.

Les propriétés dynamiques du sol se déduisent de ces résultats, pour chacun des cycles sélectionnés, avec les modules sécants en extension $E_{\mathrm{e}}$ et en compression $E_{\mathrm{c}}$, puis le module global $E_{\mathrm{t}}(2)$ et l'amortissement $D(3)$. Quand plusieurs cycles sont sélectionnés et pourvu que ceux-ci soient à peu près stabilisés, la moyenne de ces caractéristiques est associée à l'amplitude de la déformation imposée $\Delta \varepsilon_{\mathrm{a}}$ sa pendant cette séquence de cycles. La courbe de dégradation du module $E_{\mathrm{t}}$ et la courbe de l'amortissement $D$ est établie alors en fonction de toutes les amplitudes imposées d'une séquence à l'autre pendant l'essai triaxial. Les modules $E_{\mathrm{e}}$ et $E_{\mathrm{c}}$ servent à examiner la distorsion des cycles et l'écart de la réponse du sol par rapport à l'hypothèse hystérétique.

La méthode s'avère efficace pour identifier les modules d'Young sécants sur les courbes expérimentales, en particulier sous les petites amplitudes de déformation où le bruit des mesures est défavorable L'identification des amortissements 
Tableau 1. Caractéristiques moyennes d'état du limon.

Table 1. Average state characteristics of the silt.

\begin{tabular}{llllllll}
\hline$w(\%)$ & $\gamma\left(\mathrm{kN} / \mathrm{m}^{3}\right)$ & $\gamma_{\mathrm{d}}\left(\mathrm{kN} / \mathrm{m}^{3}\right)$ & $e(-)$ & $S_{\mathrm{r}}(\%)$ & $\rho_{\mathrm{d}}\left(\mathrm{g} / \mathrm{cm}^{3}\right)$ & $w_{\text {sat }}(\%)$ & $\gamma_{\mathrm{d} \mathrm{sat}}\left(\mathrm{kN} / \mathrm{m}^{3}\right)$ \\
\hline 20,08 & 19,50 & 16,24 & 0,632 & 85,9 & 1,655 & 23,39 & 20,04 \\
\hline
\end{tabular}

pendant les séquences de cycles successives est améliorée aussi. Mais cette l'application de cette méthode n'échappe pas aux questions habituelles de l'influence de la machine d'essai et des conditions d'essai (fréquence, nombre de cycles, jeu des contacts machine-éprouvette). L'identification des propriétés dynamiques du sol est accompagnée par toutes les courbes contrainte-déformation, chemins des contraintes et pressions interstitielles enregistrés pendant les séquences d'essai, afin de donner une bonne description de la réponse du sol. L'exemple qui suit en donne une illustration.

\section{Application à l'exemple d'un essai triaxial cyclique sur un limon}

L'exemple d'un essai triaxial cyclique est présenté. Il porte sur un limon testé dans son état naturel. Les propriétés dynamiques du sol sont identifiées par la méthode proposée.

\subsection{Le limon}

Le limon provient du site de Vauxrains, près de Soissons dans l'Aisne. Les échantillons carottés ont été prélevés dans trois sondages de près de cinq mètres de profondeur. Les échantillons carottés sont parfaitement bien conditionnés dans des conteneurs en PVC de $85 \mathrm{~mm}$ de diamètre intérieur, fermés à leur extrémité par des bouchons et du ruban adhésif. Ils sont stockés verticalement au laboratoire. L'ouverture des conteneurs révèle la présence d'un limon beige brun ou beige orangé, légèrement argileux ou légèrement sableux, homogène dans l'ensemble.

Les caractéristiques d'état du limon, teneurs en eau et densités, ont été mesurées sur les éprouvettes d'essai après découpage (diamètre, hauteur, masse) et en fin d'essai (masse sèche) et sur des fragments d'échantillons (plus de cent mesures de $w$ et plus de cinquante mesures de $\gamma$ ). Le Tableau 1 indique les moyennes de caractéristiques d'état où $w$ la teneur en eau, $\gamma$ le poids volumique, $\gamma_{\mathrm{d}}$ le poids volumique sec, $e$ l'indice des vides et $S_{\mathrm{r}}$ le degré de saturation.

Le poids volumique $\gamma$ et le poids volumique $\sec \gamma_{\mathrm{d}}$ sont calculés avec $g=9,81 \mathrm{~m} / \mathrm{s}^{2}$. L'indice des vides $e$ et le degré de saturation $S_{\mathrm{r}}$ sont calculés avec un poids volumique solide $\rho_{\mathrm{s}}=26,5 \mathrm{kN} / \mathrm{m}^{3}$ par défaut, soit une masse volumique solide $\rho_{\mathrm{s}}=2,70 \mathrm{~g} / \mathrm{cm}^{3}$. Le Tableau 1 indique aussi la masse volumique sèche moyenne $\rho_{\mathrm{d}}$, la teneur en eau à saturation $w_{\text {sat }}$ et le poids volumique à saturation $\gamma_{\text {sat }}$ du limon. Le sol est peu compact, avec un indice des vides moyen égal à 0,63 . Il n'est pas saturé, $S_{\mathrm{r}}<1$.

Les essais d'identification du limon comptent des analyses granulométriques par diffraction laser, des mesures des limites de consistance et des essais au bleu de méthylène. Ces propriétés sont reportées dans le Tableau $2\left(C_{2}\right.$ et $C_{80}$ teneurs en
Tableau 2. Propriétés physiques du limon.

Table 2. Physical properties of the silt.

\begin{tabular}{llllll}
\hline$C_{2}(\%)$ & $C_{80}(\%)$ & $w_{\mathrm{L}}(-)$ & $w_{\mathrm{P}}(-)$ & $I_{\mathrm{p}}(-)$ & $V_{\mathrm{B}}(\mathrm{g} / 100 \mathrm{~g})$ \\
\hline $8-16$ & $94-97$ & $26-32$ & $19-22$ & $6-12$ & $2,7-3,9$ \\
\hline
\end{tabular}

particules de diamètre inférieur à $2 \mu \mathrm{m}$ et $80 \mu \mathrm{m})$. Le sol se classe en limon peu plastique $\left(L_{\mathrm{p}}\right)$ ou argile peu plastique $\left(A_{\mathrm{p}}\right)$ d'après la classification USCS-LCPC.

\subsection{Essai triaxial à chargement monotone en compression}

Un essai triaxial à chargement monotone a été réalisé sur trois éprouvettes consolidées non drainées (diamètre $50 \mathrm{~mm}$ élancement deux). Une contre-pression de $400 \mathrm{kPa}$ est appliquée par paliers pour obtenir des coefficients $B$ de Skempton voisins ou supérieurs à 0,95 . Les trois éprouvettes sont consolidées sous des pressions effectives isotropes $p_{\mathrm{c}}$ de 50,150 et $500 \mathrm{kPa}$. Les temps de consolidation sont courts, de l'ordre de quelques dizaines de minutes, en liaison avec le caractère perméable du limon. Après consolidation, les cisaillements non drainés sont effectués en compression à des vitesses lentes $v=0,009 \mathrm{~mm} / \mathrm{min}$.

La Figure 2 montre les courbes enregistrées pendant les phases de cisaillement monotone. La Figure 2a montre les courbes contrainte-déformation $\left(\varepsilon_{\mathrm{a}}, q\right)$. Les pentes initiales des tangentes à ces courbes représentent les modules d'Young non drainés initiaux tangents $E_{\mathrm{u}}$ pour un niveau de déformation de l'ordre de $10^{-4}(93,135$ et $213 \mathrm{MPa})$. Ces modules tangents croissent avec la pression effective de consolidation $p_{\mathrm{c}}$. Par définition, ils sont supérieurs à tous les modules sécants qui les accompagnent. Après un coude marqué à moins de $1 \%$ de déformation axiale, le déviateur n'augmente plus. Le mécanisme d'écrouissage est inexistant, ou très limité à basse pression. La déformation axiale est poussée à plus de $20 \%$. Les éprouvettes se déforment en tonneau.

Sur la Figure $2 b$, les chemins des contraintes effectives sont indiqués dans le plan des contraintes effectives $(p, q)$. Ils sont dirigés vers les pressions $p$ décroissantes pour $p_{\mathrm{c}}>150 \mathrm{kPa}$ et dans l'autre direction à basse pression. Le sol est légèrement surconsolidé à basse pression, puis normalement consolidé audelà. Les essais œdométriques réalisés sur le limon donnent des pressions effectives de préconsolidation de cet ordre $\left(\sigma^{\prime}{ }_{\mathrm{a}} \approx 150 \mathrm{kPa}\right)$. Ce comportement est typique d'un sol fin peu argileux. Une interprétation «moyenne » est néanmoins proposée en contraintes effectives avec une cohésion $c^{\prime}=4 \mathrm{kPa}$ et un angle de frottement $\varphi^{\prime}=31,7^{\circ}$. Ces caractéristiques de résistance sont tirées des propriétés de la droite de Coulomb 

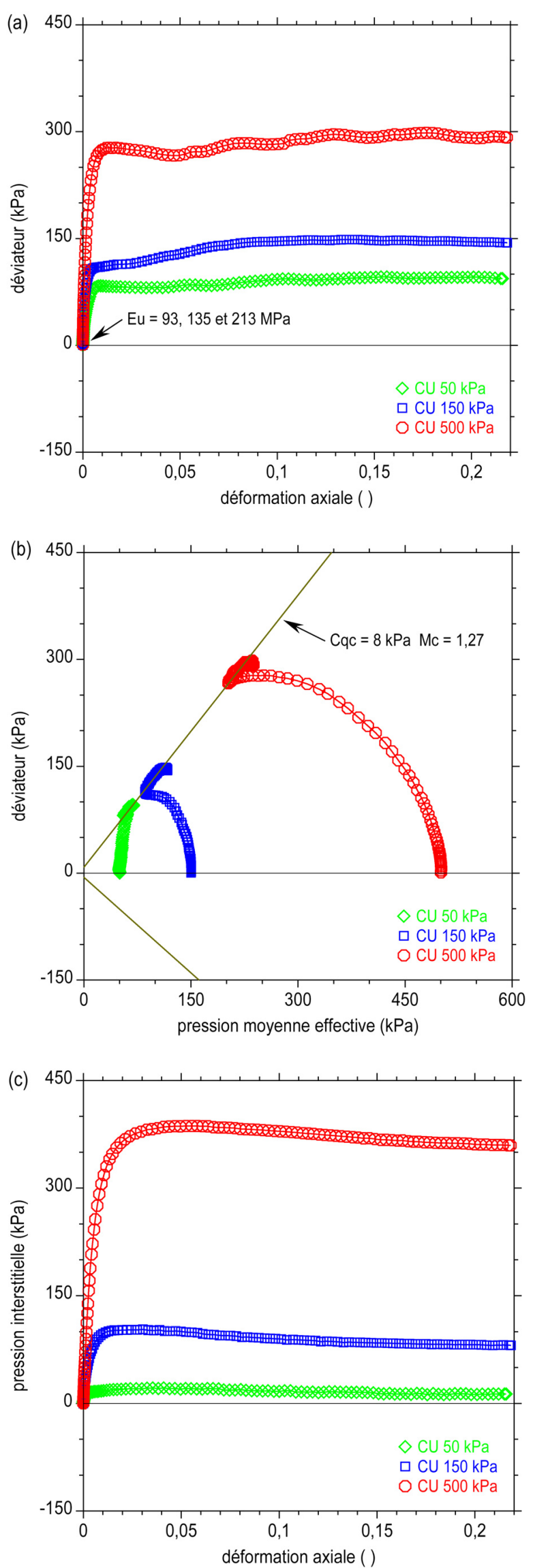

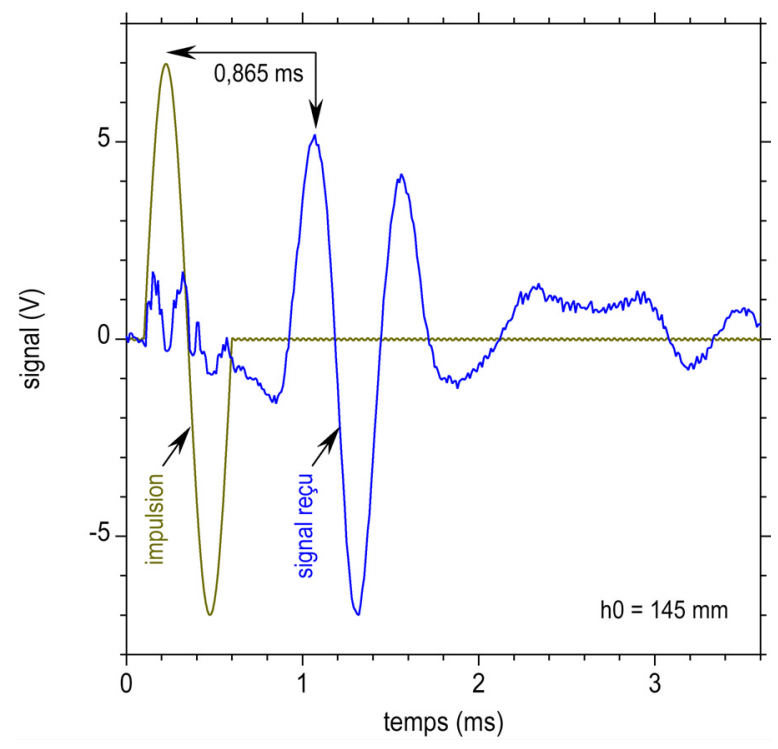

Fig. 3. Mesure de la vitesse des ondes de cisaillement au triaxial sur le limon. Impulsion donnée et signal reçu par les cellules piézoélectriques.

Fig. 3. Shear wave velocity measurement in the triaxial cell on the silt. Impulse and recorded signal by the bender elements.

exprimée dans le plan $(p, q)$, de pente $M_{\mathrm{c}}=1,27$ et d'ordonnée à l'origine $C_{\mathrm{qc}}=8 \mathrm{kPa}$. Les pressions interstitielles sont représentées en fonction de la déformation axiale $\left(\varepsilon_{\mathrm{a}}, u\right)$ sur la Figure 2c.

\subsection{Vitesse des ondes de cisaillement}

La vitesse des ondes de cisaillement $V_{\mathrm{s}}$ a été mesurée sur une éprouvette triaxiale de limon. La cellule est instrumentée à l'aide d'un couple de cellules piézo-électriques, ou « bender element », disposées au centre des plaques poreuses de pied et de tête (Shirley et Hampton, 1978 ; Dyvik et Madshus, 1986).

La Figure 3 montre un enregistrement réalisé où le signal émis et le signal reçu sont représentés en fonction du temps. La distance entre les cellules piézo-électriques est égale à la hauteur de l'éprouvette $h_{0}=145 \mathrm{~mm}$. Le temps de trajet de l'onde de cisaillement est pointé entre les deux premiers pics, soit $0,865 \mathrm{~ms}$. La vitesse $V_{\mathrm{s}}$ des ondes de cisaillement ainsi mesurée vaut $168 \mathrm{~m} / \mathrm{s}$. Le module de cisaillement maximal $G_{\max }$ du limon est lié à la vitesse $V_{\mathrm{s}}$ par la relation $G_{\max }=\rho V_{\mathrm{s}}^{2}$, où $\rho$ est la masse volumique du limon. Avec $\rho=1,93 \mathrm{~g} / \mathrm{cm}^{3}$ pour cette éprouvette et $V_{\mathrm{s}}=168 \mathrm{~m} / \mathrm{s}$, le module $G_{\max }$ du limon vaut $G_{\max }=54 \mathrm{MPa}$.

Fig. 2. Essai triaxial non drainé monotone en compression. a) Courbes contrainte-déformation dans le plan $\left(\varepsilon_{\mathrm{a}}, q\right)$. b) Chemins des contraintes effectives dans le plan $(p, q)$. c) Courbes pression interstitielle-déformation dans le plan $\left(\varepsilon_{\mathrm{a}}, u\right)$.

Fig. 2. Undrained monotonic compression triaxial test. a) Stress strain curves $\left(\varepsilon_{a}, q\right)$. b) Effective stress paths $(p, q)$. c) Pore pressure versus axial strain $\left(\varepsilon_{a}, u\right)$. 
Tableau 3. Amplitudes des séquences de cycles et récapitulation des propriétés dynamiques du limon.

Table 3. Cyclic strain amplitudes and summary of the dynamics properties of the silt.

\begin{tabular}{lllllr}
\hline Séquence de cycles & $\Delta \varepsilon_{\mathrm{a} \mathrm{SA}}(-)$ & $\Delta q_{\mathrm{SA}}(\mathrm{kPa})$ & $E_{\mathrm{e}}(\mathrm{MPa})$ & $E_{\mathrm{c}}(\mathrm{MPa})$ & $\left.E_{\mathrm{t}} \mathrm{MPa}\right)$ \\
\hline$y_{1}$ & 0,000051 & 3,4 & 59,3 & 72,2 & 65,9 \\
$y_{2}$ & 0,000102 & 6,1 & 62,6 & 55,9 & 59,2 \\
$y_{3}$ & 0,000203 & 10,1 & 53,7 & 46,5 & 0,000 \\
$y_{4}$ & 0,00051 & 17,6 & 38,0 & 31,2 & 0,007 \\
$y_{5}$ & 0,00102 & 23,8 & 26,8 & 20,1 & 34,6 \\
$y_{6}$ & 0,00203 & 30,6 & 17,5 & 12,7 & 23,3 \\
$y_{7}$ & 0,051 & 31,2 & 7,36 & 5,00 & 0,034 \\
$y_{8}$ & 0,102 & 29,5 & 3,44 & 2,38 & 0,071 \\
\hline
\end{tabular}

\subsection{Essai triaxial cyclique, courbes expérimentales}

Un essai triaxial CU cyclique a été réalisé en mode DF, essai contrôlé en déplacement et en fréquence, sur le limon. L'éprouvette de $50 \mathrm{~mm}$ de diamètre et d'élancement deux est saturée sous contre-pression $\left(u_{\mathrm{cp}}=400 \mathrm{kPa}\right)$, puis elle est consolidée sous la pression effective isotrope $p_{\mathrm{c}}=50 \mathrm{kPa}$. Les cycles en mode DF sont appliqués ensuite en condition non drainée à la fréquence $f=0,5 \mathrm{~Hz}$ (période $T=2 \mathrm{~s}$ ). Huit séquences de cinquante cycles ont été appliquées pour des amplitudes $\Delta \varepsilon_{\mathrm{a}} \mathrm{SA}$ égales à $0,005 \%, 0,01 \%, 0,02 \%, 0,05 \%$, $0,1 \%, 0,2 \%, 0,5 \%$ et $1 \%$ respectivement, notées $y_{1}$ à $y_{8}$ comme indiqué dans le Tableau 3.

En cours d'essai et pendant les quatre premières séquences de cycles $y_{1}$ à $y_{4}$, les enregistrements montrent que l'accroissement de la pression interstitielle reste limité (inférieur à $5 \mathrm{kPa}$ ). Elles ont été enchaînées l'une après l'autre de ce fait, jusqu'à la séquence $y_{5}$. Des phases de reconsolidation sont effectuées ensuite avant les séquences de cycles $y_{6}, y_{7}$ et $y_{8}$, puis après la séquence $y_{8}$. Les premières produisent des déformations volumiques cumulées limitées, inférieures à $0,5 \%$. Par contre, les séquences de cycles $y_{6}, y_{7}$ et $y_{8}$ provoquent des montées de pression interstitielle plus fortes $(17,25$ et $35 \mathrm{kPa})$, puis des déformations volumiques de reconsolidation cumulées de près de $0,5,1$ et $2 \%$.

Les courbes contrainte-déformation $\left(\varepsilon_{\mathrm{a}}, q\right)$ des huit séquences de cycles sont rassemblées sur les deux graphiques de la Figure 4, en séparant les quatre premières séquences des quatre suivantes avec des échelles appropriées. Pendant les premières séquences de cycles, les courbes forment des boucles très étroites et fermées, alors que les dernières se développent largement et ne sont pas fermées. Les modules d'Young sécants se stabilisent rapidement pendant les premières séquences et ne se stabilisent pas pendant les dernières. Ils diminuent d'une séquence à l'autre. Ces évolutions caractérisent la dégradation du module d'Young $E_{\mathrm{u}}$ en fonction de l'amplitude $\Delta \varepsilon_{\mathrm{a}} \mathrm{SA}$ des séquences successives de cycles. Parallèlement, les aires intérieures des boucles $\left(\varepsilon_{\mathrm{a}}, q\right)$ croissent avec $\Delta \varepsilon_{\mathrm{a}}$ SA, marquant ainsi une dissipation de plus en plus grande, ce qui sera traduit par l'accroissement de l'amortissement $D$.

Les graphiques de la Figure 5 montrent les enregistrements effectués pendant la séquence de cycles $y_{6}(50$ cycles, période $T=2 \mathrm{~s}$ ). La déformation axiale $\varepsilon_{\mathrm{a}}$ est représentée en fonction du temps sur la Figure 5a. Son amplitude imposée $\Delta \varepsilon_{\mathrm{a}}$ sA est égale à $0,2 \%$. En dessous (Fig. $5 \mathrm{c}$ ), le déviateur est alterné,
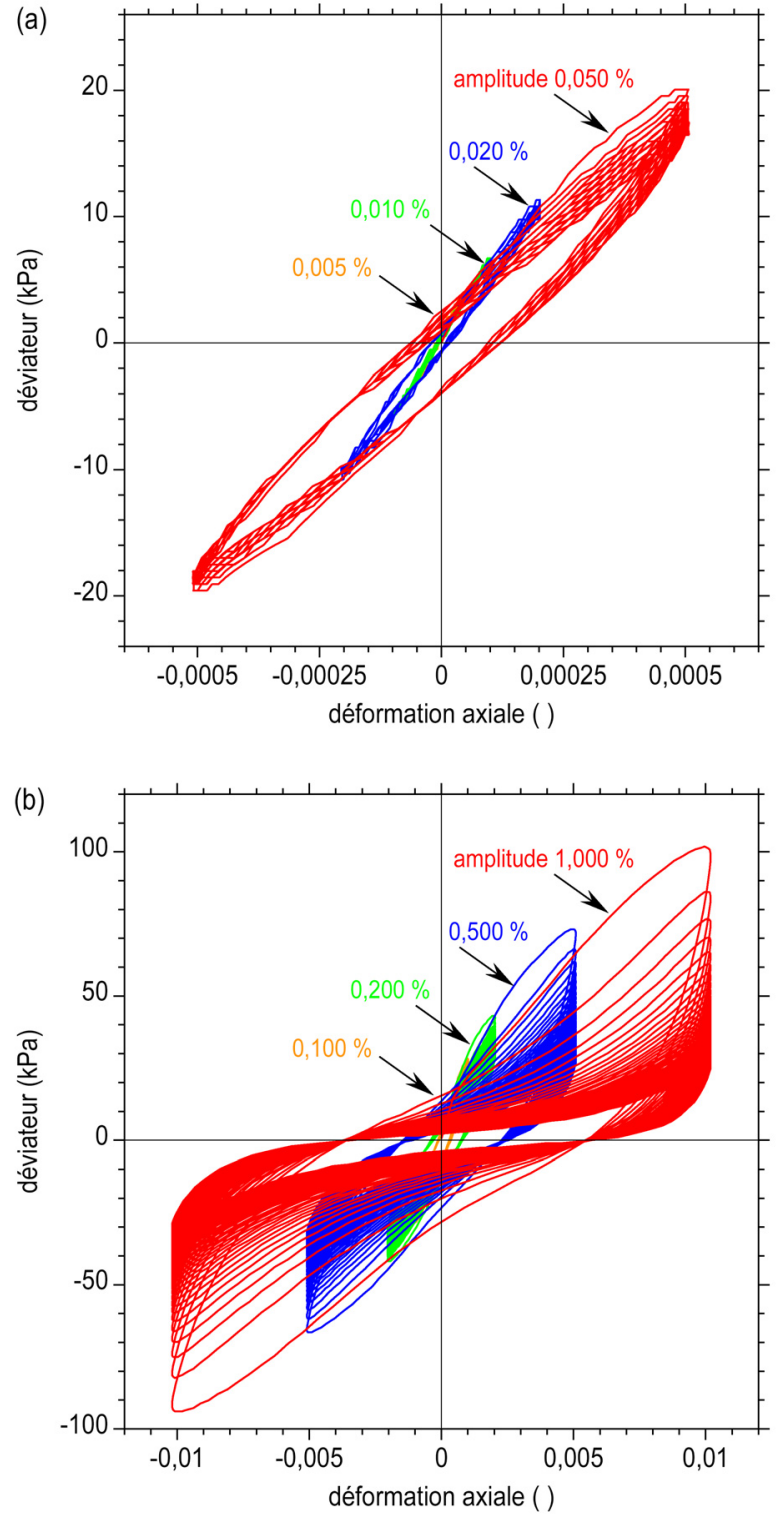

Fig. 4. Courbes contrainte-déformation $\left(\varepsilon_{\mathrm{a}}, q\right)$. a) Séquences de cycles $y_{1}$ à $y_{4}$. b) Séquences de cycles $y_{5}$ à $y_{8}$.

Fig. 4. Deviator stress versus axial strain curves $\left(\varepsilon_{a}, q\right)$. a) Sequences of cycles $y_{1}$ to $y_{4}$. b) Sequences of cycles $y_{5}$ to $y_{8}$. 

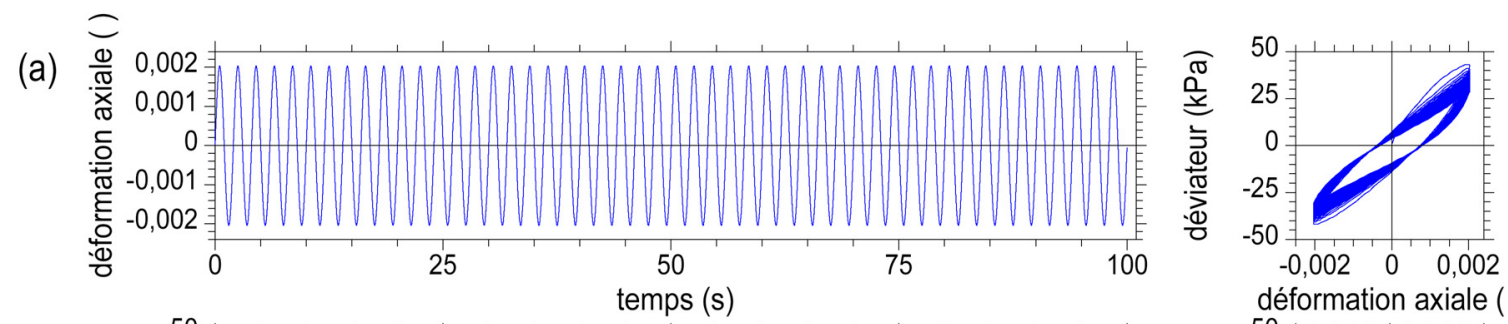

(b)

(c)
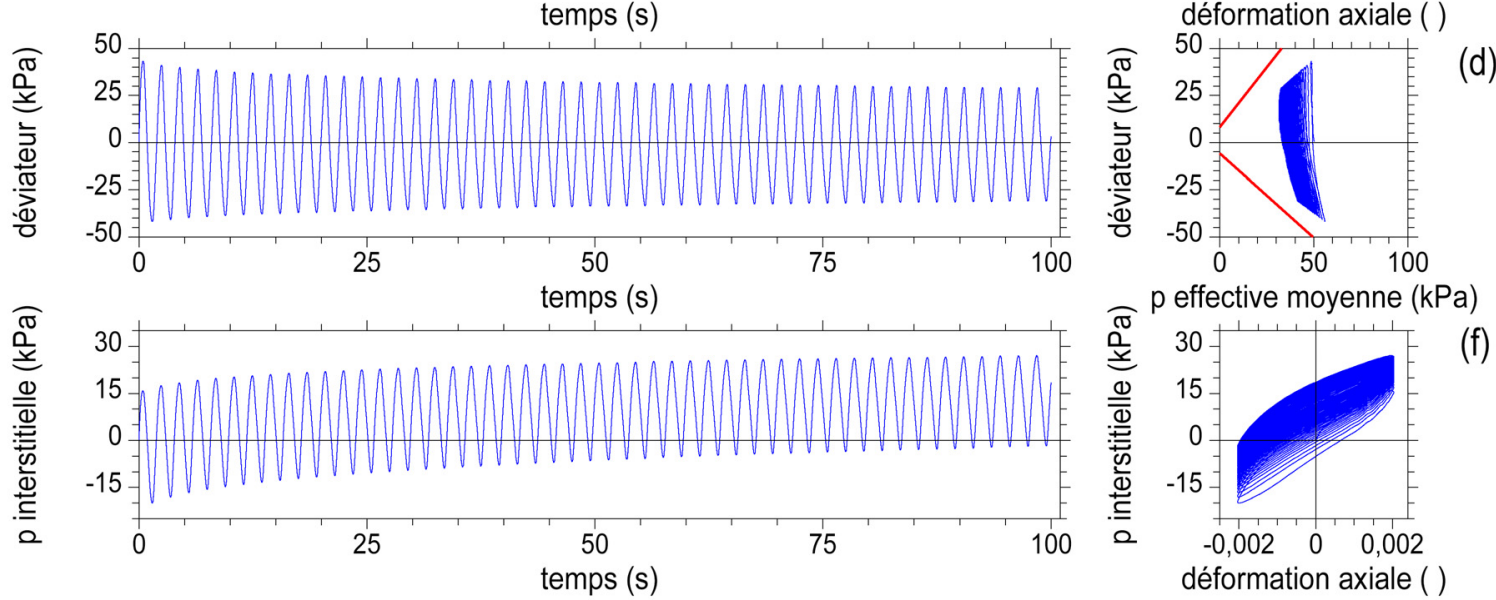

Fig. 5. Courbes enregistrées pendant la séquence de cycles $y_{6}$. a) Déformation axiale en fonction du temps $\left(t, \varepsilon_{\mathrm{a}}\right)$. b) Courbe contrainte-déformation $\left(\varepsilon_{\mathrm{a}}, q\right)$. c) Déviateur en fonction du temps $(t, q)$. d) Chemin des contraintes effectives $(p, q)$. e) Pression interstitielle en fonction du temps $(t, u)$. f) Pression interstitielle en fonction de la déformation axiale $\left(\varepsilon_{\mathrm{a}}, u\right)$.

Fig. 5. Curves recorded during the sequence $y_{6}$. a) Axial strain versus time $\left(t, \varepsilon_{a}\right)$. b) Stress-strain curve $\left(\varepsilon_{a}\right.$, q). c) Deviator stress versus time $(t, q)$. d) Effective stress path $(p, q)$. e) Pore pressure versus time $(t, u)$. f) Pore pressure versus axial strain $\left(\varepsilon_{a}, u\right)$.

successivement positif (compression) et négatif (extension). Son amplitude diminue au début de la séquence de cycles, puis cette progression s'atténue selon une loi logarithmique du temps. Sur la Figure 5e, la pression interstitielle croît rapidement en moyenne au début de la séquence de cycles, puis sa progression s'amortit mais ne cesse pas. La pression interstitielle cumulée atteint $17 \mathrm{kPa}$ à la fin des cinquante cycles $\left(34 \%\right.$ de $p_{\mathrm{c}}$ ). Une phase de reconsolidation s'impose avant de passer à la séquence de cycles $y_{7}$.

Le graphique de la Figure $5 \mathrm{~b}$ montrent la courbe contrainte-déformation $\left(\varepsilon_{\mathrm{a}}, q\right)$ pendant la séquence de cycles $y_{6}$. Les boucles s'infléchissent progressivement (adaptation). Au-dessous, Figure 5d, le plan $(p, q)$ montre le chemin des contraintes effectives suivi depuis la fin de la consolidation isotrope du limon. Les deux droites indiquées représentent les enveloppes de Coulomb de compression et d'extension déduites de l'essai triaxial non drainé monotone (Fig. 2). Les cycles se situent encore à une distance éloignée des critères à ce stade de l'essai. Enfin, le graphique de la Figure 5 f montre l'accumulation progressive de la pression interstitielle avec les cycles, en fonction de la déformation axiale.

En pratique, seule la courbe contrainte-déformation $\left(\varepsilon_{\mathrm{a}}, q\right)$ est utilisée pour identifier les propriétés dynamiques du sol (Fig. 5b). Les autres courbes sont indiquées en vue de compléter la description de la réponse du sol. Toutefois, l'intérêt de ces courbes dépend de la validité et de la qualité de la mesure de la pression interstitielle (Fig. 5d, e et f). À ce titre, le calcul élémentaire suivant permet de comparer les vitesses de chargement cycliques et monotones. Au cours des cycles non drainés pilotés en mode déplacement, la sollicitation est sinusoïdale de période $T$ et d'amplitude $\Delta \varepsilon_{\mathrm{a} \mathrm{SA}}$,
$\varepsilon_{\mathrm{a}}=\Delta \varepsilon_{\mathrm{a} \mathrm{SA}} \sin (2 \pi t / T)$. La vitesse de chargement maximale vaut $2 \pi \varepsilon_{\mathrm{a} \mathrm{SA}} / T$. Ainsi, pour une amplitude $\Delta \varepsilon_{\mathrm{a} \mathrm{SA}}=0,001$ $(0,1 \%)$ et une période $T=2 \mathrm{~s}$, la vitesse maximale de déformation est $2 \pi \times 0,001 / 2=0,0031 / \mathrm{s}=3,110^{-03} / \mathrm{s}$. En comparaison, la vitesse de déformation d'un essai monotone tel que celui qui est présenté sur la Figure 2 est de 0,009/ $(60 \times 100)=1,510^{-6} / \mathrm{s}$, pour une vitesse de chargement $v=0,009 \mathrm{~mm} / \mathrm{min}$ et une éprouvette de $100 \mathrm{~mm}$ de hauteur. Le rapport des vitesses est de 2000 dans le cas particulier des essais évoqués ici. Ainsi, les chargements cycliques effectués aux fréquences habituelles sont plus rapides que les chargements monotones, ce qui ne fournit pas les meilleures conditions de mesure de la pression interstitielle et ne favorise pas l'interprétation de l'essai en contraintes effectives.

\subsection{Exploitation de l'essai triaxial cyclique}

Dans la méthode proposée, les courbes représentant la déformation $\left(t, \varepsilon_{\mathrm{a}}\right)$ et le déviateur $(t, q)$ en fonction du temps font l'objet d'une approximation à l'aide de séries de Fourier. Ces approximations sont calculées au sens des moindres carrés sur la totalité de la séquence de cycles, ou un intervalle particulier de celle-ci. Dans le cas présent, le calcul est effectué sur les dix derniers cycles de chacune des séquences $y_{1}$ à $y_{8}$, soit entre 80 et $100 \mathrm{~s}$ après le début de la sollicitation sinusoïdale (Fig. 5). Cela permet de sélectionner un domaine où l'amplitude $\Delta q$ du déviateur est la plus amortie et les boucles $\left(\varepsilon_{\mathrm{a}}, q\right)$ les mieux fermées. Parallèlement, l'accroissement de la pression interstitielle est plus lent dans cet intervalle. D'autres choix d'intervalles de calcul sont possibles, comme évoqué plus haut. 
(a)

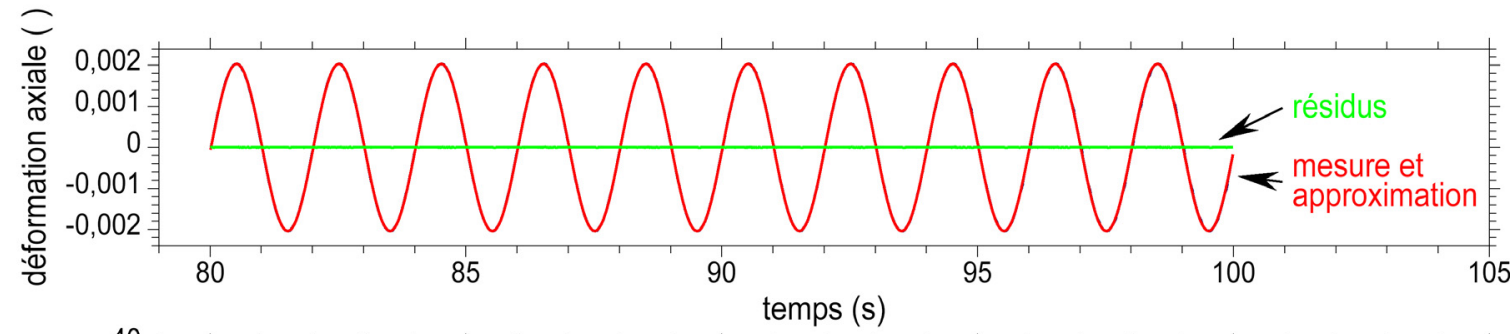

(b)

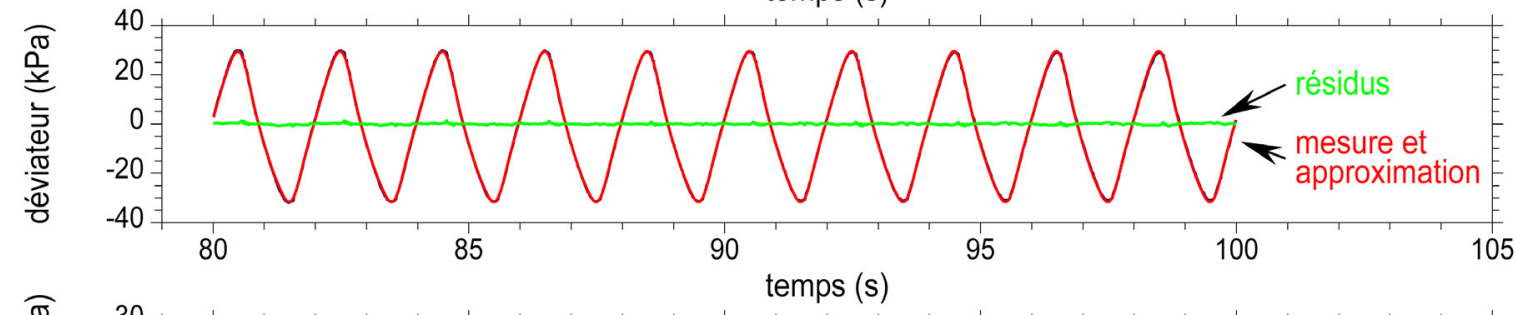

(c)

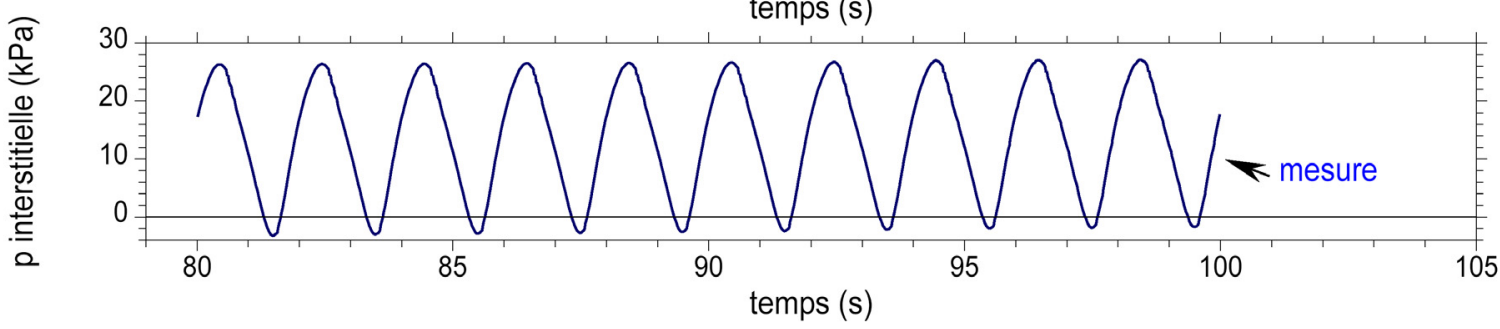

Fig. 6. Courbes enregistrées pendant les cycles 41 à 50 de la séquence $y_{6}$ et approximations. a) Déformation axiale en fonction du temps $\left(t, \varepsilon_{\mathrm{a}}\right)$. b) Déviateur en fonction du temps $(t, q)$. d) Pression interstitielle en fonction du temps $(t, u)$.

Fig. 6. Curves recorded during the cycles 41 to 50 of the sequence $y_{6}$ and approximations. a) Axial strain versus time ( $t$, $\left.\varepsilon_{a}\right)$. b) Deviator stress versus time $(t, q)$. e) Pore pressure versus time $(t, u)$.

La courbe $\left(t, \varepsilon_{\mathrm{a}}\right)$, qui représente la sollicitation, est décrite par une seule fréquence en principe, celle qui a été imposée, ici $f=0,5 \mathrm{~Hz}$. Néanmoins, le bruit des mesures doit être éliminé aussi, ce qui justifie que la méthode soit appliquée à ce signal. De même et concernant le déviateur, qui constitue la réponse du limon, le bruit est éliminé par filtrage, pour ne retenir que les fréquences dominantes de la courbe calculée $(t, q)$. Les résidus, c'est-à-dire les écarts entre la courbe expérimentale et la courbe calculée, sont précisés point par point. Un exemple est donné sur les graphiques de la Figure 6 où sont représentées les courbes enregistrées pendant les cycles 41 à 50 et les approximations. Sur la Figure 6a, la déformation axiale mesurée, qui est aussi la sollicitation, est donnée en fonction du temps $\left(t, \varepsilon_{\mathrm{a}}\right)$. Son approximation se superpose parfaitement et les résidus sont nuls. Sur la Figure 6b, le déviateur mesuré (réponse) et son approximation sont représentés en fonction du temps $(t, q)$. Les courbes sont quasi superposées. Leur forme s'éloigne d'une sinusoïde. Les résidus ne sont pas totalement nuls. La méthode proposée consiste à éliminer ce bruit afin d'obtenir une identification plus précise des modules et de l'amortissement. La pression interstitielle est indiquée ensuite en fonction du temps, mais elle ne fait pas l'objet d'une approximation ici (Fig. 6c). Connaissant les approximations $\varepsilon_{\mathrm{a}}(t)$ et $q(t)$ dans l'intervalle sélectionné de la séquence des cycles, la courbe contrainte-déformation $\left(\varepsilon_{\mathrm{a}}, q\right)$ peut être établie alors. Les caractéristiques dynamiques décrites sur la Figure 1 sont identifiées ensuite. Puis la méthode d'identification des paramètres dynamiques du limon est appliquée aux séquences $y_{1}$ à $y_{8}$ de l'essai triaxial cyclique. Ces paramètres sont reportés dans le Tableau 3. Les modules $E_{\mathrm{e}}$ et $E_{\mathrm{c}}$ encadrent le module $E_{\mathrm{t}}$. L'évolution constatée dans cet exemple n'est pas systématique et change d'un sol à l'autre ou dépend des conditions d'essai. L'écart entre les modules souligne la distorsion des cycles.

Les Figures 7 et 8 montrent les courbes contraintedéformation $\left(\varepsilon_{\mathrm{a}}, q\right)$ enregistrées pendant les dix derniers cycles des séquences $y_{3}$ à $y_{5}$ et $y_{6}$ à $y_{8}$, puis les approximations correspondantes. Les courbes expérimentales et les approximations sont représentées sur des graphiques séparés pour montrer l'effet du lissage opéré par la méthode proposée. La règle de fermeture des boucles $\left(\varepsilon_{\mathrm{a}}, q\right)$ est vérifiée pour toutes les séquences de cycles Avec les approximations sont figurés par un point les centres des cycles $\left(\varepsilon_{\mathrm{a} 0}, q_{0}\right)$, puis les segments de droite de compression et d'extension dont les pentes représentent les modules d'Young sécants $E_{\mathrm{c}}$ et $E_{\mathrm{e}}$. Ces deux modules sécants ainsi que le module global sécant $E_{\mathrm{t}}$ sont calculés. L'aire du cycle $A_{\mathrm{c}}$ puis l'aire du triangle $A_{\mathrm{t}}$ sont calculées, pour donner l'amortissement $D$.

Les courbes contrainte-déformation $\left(\varepsilon_{\mathrm{a}}, q\right)$ enregistrées et calculées des Figures 7 et 8 montrent bien l'évolution de la forme des cycles en fonction l'amplitude $\Delta \varepsilon_{\mathrm{a}}$ SA de déformation imposée. Seules les boucles des trois premières séquences de cycles conservent l'allure d'une réponse hystérétique (Fig. 2). À partir de la séquence $y_{4}$, où $\Delta \varepsilon_{\mathrm{a} \mathrm{SA}}=0,0005=$ $0,05 \%$, les boucles prennent une forme en « $\mathrm{S}$ inversé » de plus en plus prononcée. Ce type de réponse s'observe le plus souvent. De telles boucles d'hystérésis ne peuvent pas être modélisées sur la base de la règle de Masing. Á ce titre, Nogami et al. (2012) proposent un modèle de huit paramètres qui permet de décrire des boucles d'hystérésis en forme en 

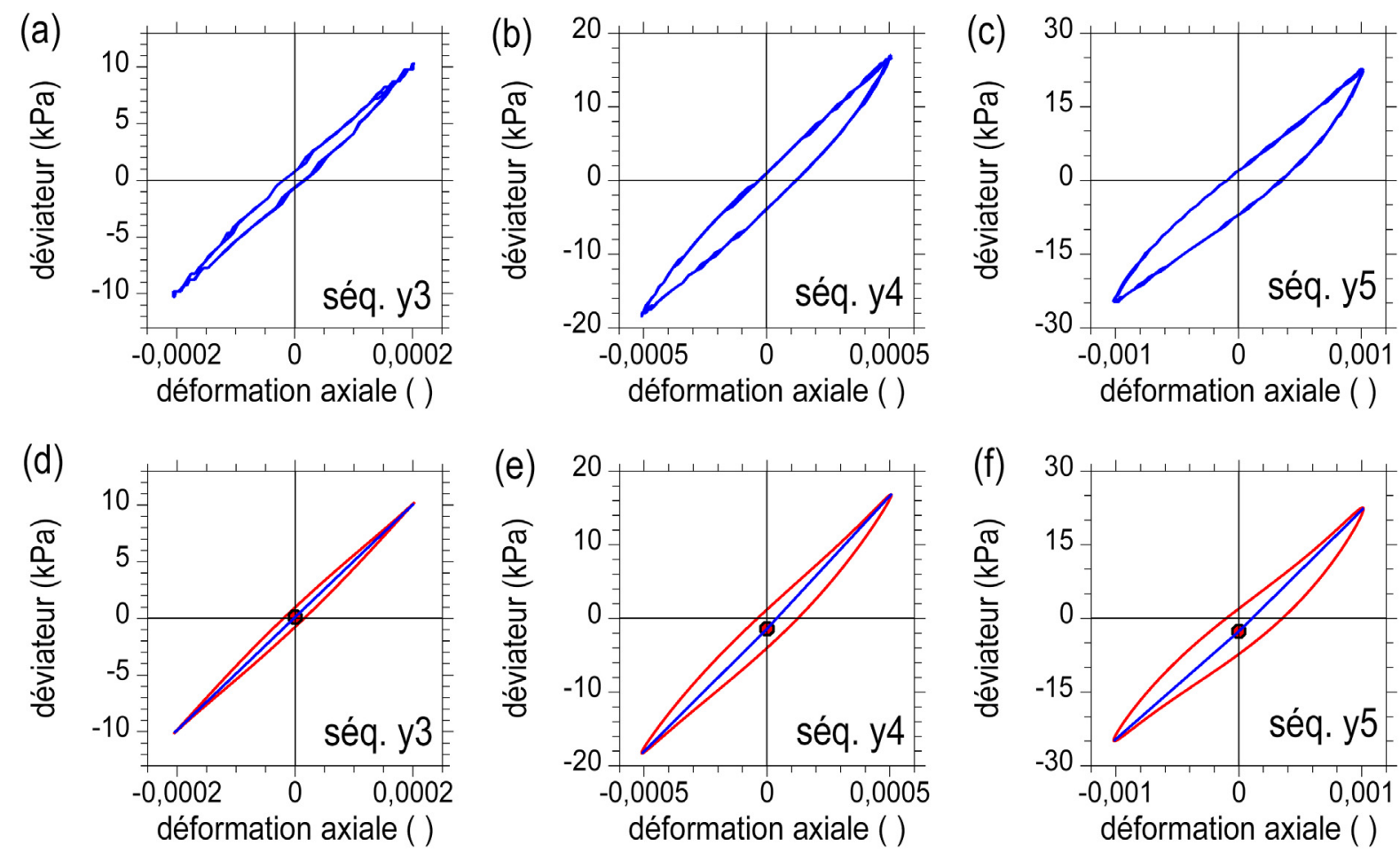

Fig. 7. Courbes contrainte-déformation $\left(\varepsilon_{\mathrm{a}} q\right)$ enregistrées pendant les dix derniers cycles des séquences $y_{3}$ à $y_{5}$. a-c) Courbes expérimentales pour les amplitudes $\Delta \varepsilon_{\mathrm{a}} \mathrm{SA}=0,0002,0,0005$ et 0,001 . d-f) Approximations correspondantes.

Fig. 7. Stress-strain curves $\left(\varepsilon_{a} q\right)$ recorded during the last 10 cycles of sequences $y_{3}$ to $y_{5}$. a-c) Experimental curves for amplitudes $\Delta \varepsilon_{a S A}=0,0002,0,0005$ et 0,001. $\left.d-f\right)$ Corresponding approximations.
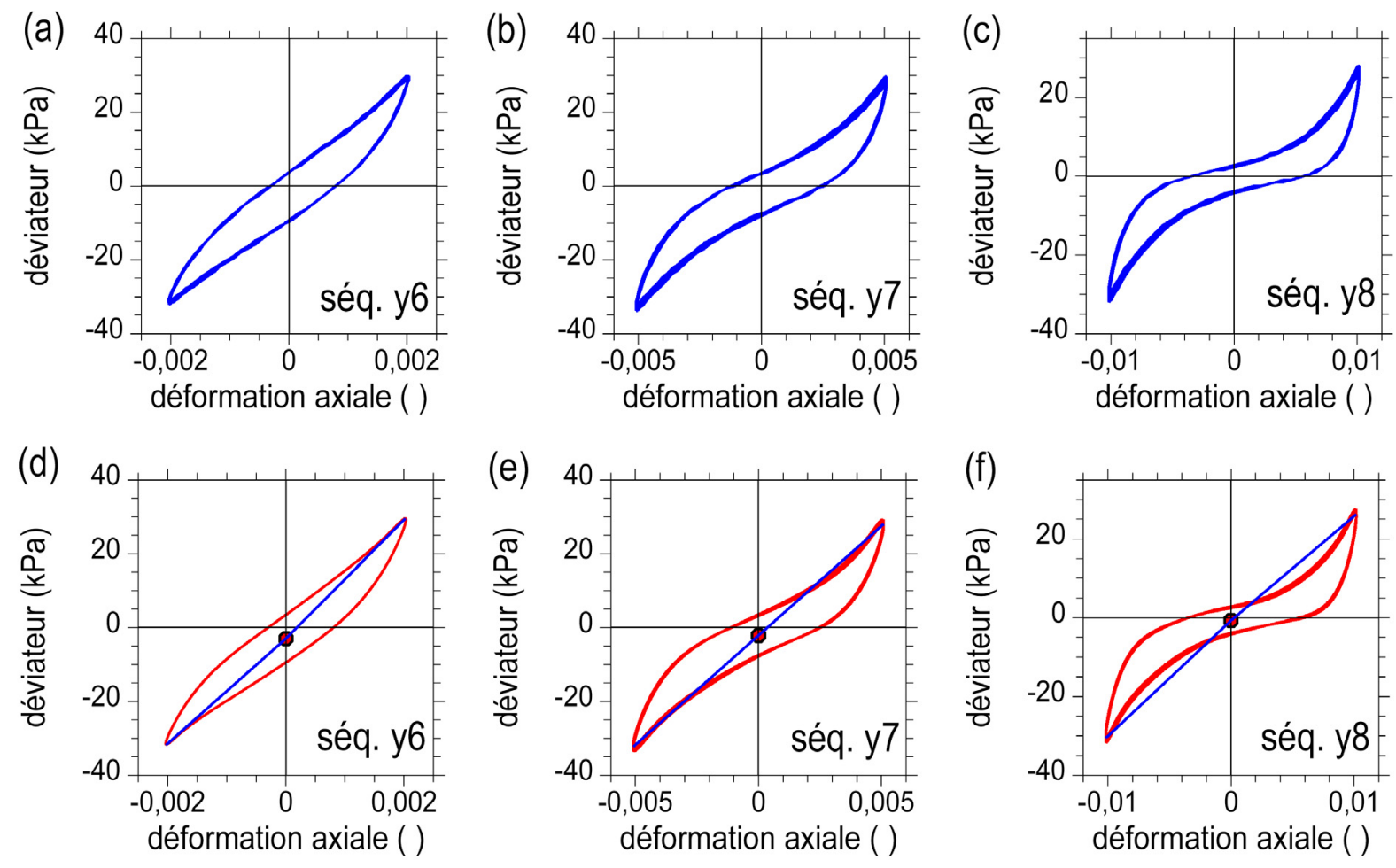

Fig. 8. Courbes contrainte-déformation $\left(\varepsilon_{\mathrm{a}} q\right)$ enregistrées pendant les dix derniers cycles des séquences $y_{6}$ à $y_{8}$. a-c) Courbes expérimentales pour les amplitudes $\Delta \varepsilon_{\mathrm{a} \text { SA }}=0,002,0,005$ et 0,01 . d-f) Approximations correspondantes.

Fig. 8. Stress-strain curves $\left(\varepsilon_{a} q\right)$ recorded during the last ten cycles of sequences $y_{6}$ to $y_{8}$. a-c) Experimental curves for amplitudes $\Delta \varepsilon_{a S A}=0,002,0,005$ et $\left.0,01 . d-f\right)$ Corresponding approximations. 
« $\mathrm{S}$ inversé ». Les paramètres sont à identifier sur les courbes d'essais cycliques. Les auteurs proposent des valeurs usuelles indicatives de ces paramètres.

Sous les plus grandes amplitudes de la déformation axiale, les boucles se distordent, les cycles ne sont plus fermés, des déformations plastiques s'accumulent. Cette évolution est progressive pour les sols fins comme ce limon ou les argiles. Elle peut être plus marquée dans les sables, voire brutale dans les sables lâches qui se liquéfient. Les amortissements semblent atteindre une limite avec les grandes amplitudes des déformations, voire une régression comme indiqué dans le Tableau 3, en relation avec l'effilement de la boucle d'hystérésis pendant la séquence de cycles $y_{8}$ ici, Figure 8c et f. Nogami et al. (2012) soulignent aussi de telles observations.

\subsection{Représentation de la réponse dynamique du limon}

Pour terminer, les paramètres dynamiques du limon, qui ont été mesurés à partir de l'essai triaxial cyclique non drainé présenté ici, sont comparés aux propriétés tirées de deux formulations empiriques. Les modules sécants $E_{\mathrm{t}}$ et les amortissements $D$ du Tableau 3 sont reportés sur les graphiques de la Figure 9 en fonction de l'amplitude de la déformation axiale $\Delta \varepsilon_{\mathrm{a}} \mathrm{SA}$.

Sur la base d'une compilation de nombreux essais de laboratoire publiés dans la littérature concernant des argiles et des limons, Vardanega et Bolton (2013) ont proposé une expression semi-empirique donnant le module maximal de cisaillement $G_{\max }$ en fonction de la pression effective de confinement et de l'indice des vides. Le module de cisaillement $G$ s'exprime, via le rapport $G / G_{\max }$, en fonction de la déformation de cisaillement $\gamma$ et d'une déformation de référence $\gamma_{\text {ref. }}$ Comme $G_{\max }$ pour l'axe des modules $G$ en ordonnée, $\gamma_{\text {ref }}$ est introduite là pour normaliser l'axe des déformations en abscisse. Son expression est établie en fonction de l'indice de plasticité $I_{\mathrm{p}}$ :

$$
\gamma_{\text {ref }}=2,2\left(I_{\mathrm{p}} / 100000\right) \text {. }
$$

Le rapport $G_{\max } / G$ est donné alors par l'expression :

$$
G_{\max } / G=1+\left(\gamma / \gamma_{\text {ref }}\right)^{0,943}
$$

Cette formulation établie en termes de cisaillement est convertie dans les variables triaxiales $\varepsilon_{\mathrm{a}}$ et $q$ en admettant que $E_{\mathrm{u}}=1,5 \mathrm{G}$ et que $\gamma=1,5 \varepsilon_{\mathrm{a}}$. Il est admis aussi que le module d'Young non drainé maximal vaut $E_{\mathrm{u} \text { max }}=80 \mathrm{MPa}$ d'après la vitesse des ondes de cisaillement $V_{\mathrm{s}}$ mesurée sur le limon. Les courbes obtenues ainsi sont reportées dans le plan $\left(\Delta \varepsilon_{\mathrm{a}} \mathrm{SA}, E_{\mathrm{u}}\right)$ de la Figure 9a pour des indices de plasticité allant de 10 à 200 . Les points expérimentaux tirés de l'essai triaxial cyclique (Tab. 3) sont reportés sur le graphique. Ces points sont groupés autour des courbes empiriques $I_{\mathrm{p}}=10$ et 15 qui reflètent à peu près l'indice de plasticité du limon (Tab. 2).

Comme évoqué plus haut, différents auteurs ont proposé de relier l'amortissement $D$ au rapport $G / G_{\max }$ au moyen de formulations empiriques appropriées (Hardin et Drnevich, 1972a ; Ishibashi et Zhang, 1993). La relation polynomiale en $G / G_{\max }$ de ces derniers est mise en œuvre ici. Elle est
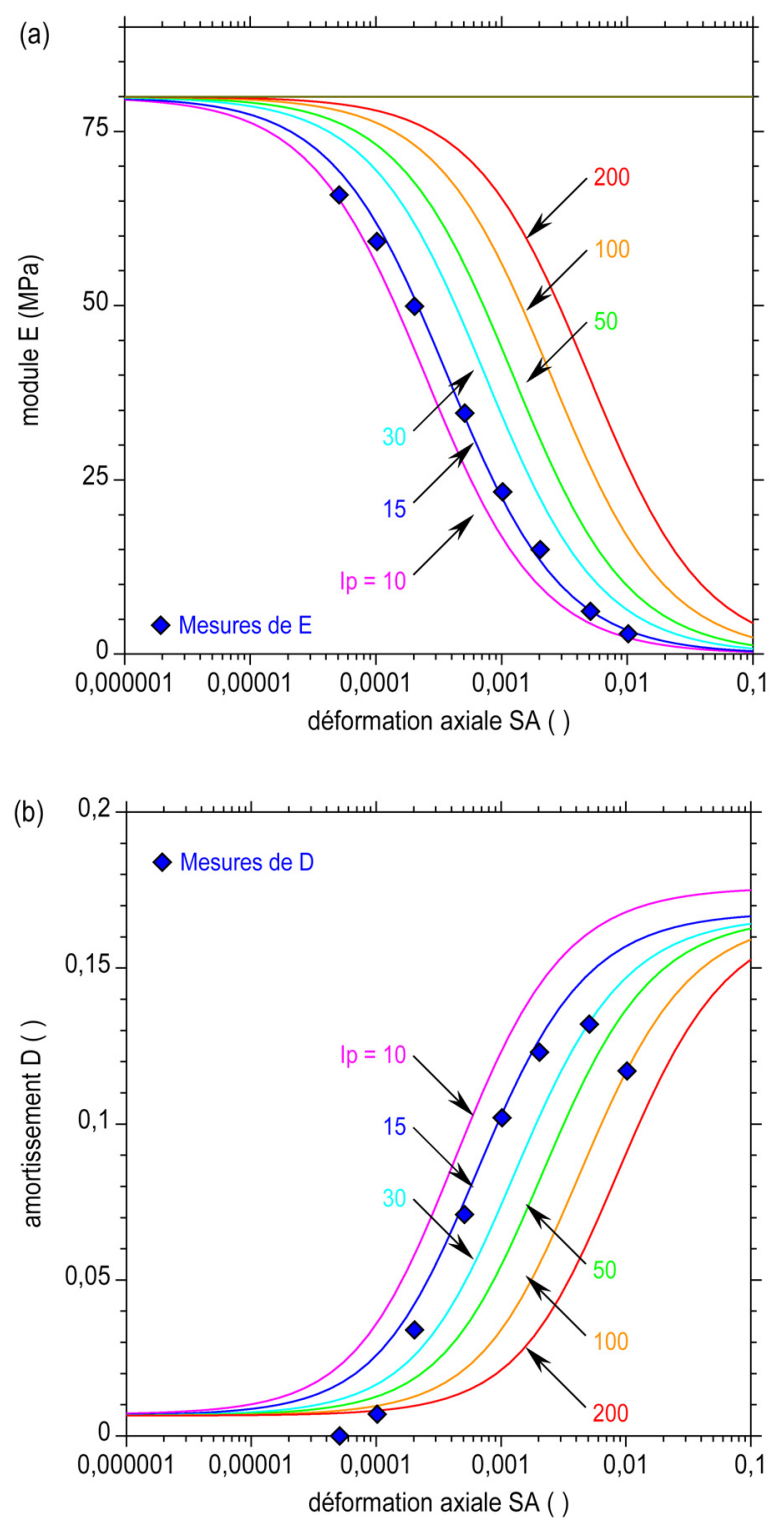

Fig. 9. Propriétés dynamiques du limon en fonction de l'amplitude $\Delta \varepsilon_{\mathrm{a}}$ SA. Comparaison des données expérimentales aux formulations empiriques. a) Modules d'Young $E_{\mathrm{u}}$. b) Amortissements $D$.

Fig. 9. Dynamic properties of the silt versus amplitude $\Delta \varepsilon_{a} S A$. Comparison of experimental data with empirical formulations. a) Young modulus $E_{u}$. b) Damping $D$.

paramétrée aussi en fonction de l'indice de plasticité $I_{\mathrm{p}}$. Elle est constituée de deux termes :

$$
\begin{gathered}
D_{I_{\mathrm{p}}}=0,167\left[1+\exp \left(-0,0145\left(I_{\mathrm{p}}\right)^{1,3}\right)\right], \\
D=D_{I_{\mathrm{p}}}\left[0,586\left(G / G_{\max }\right)^{2}-1,546\left(G / G_{\max }\right)+1\right] .
\end{gathered}
$$

Dans ces expressions, l'amortissement minimal pour $\gamma=0$ est $D_{\min }=0,039 D_{I \mathrm{p}}$, tandis que l'amortissement maximal pour $\gamma=\propto$ est $D_{\alpha}=D_{I \mathrm{p}}$. Les courbes calculées par ces expressions sont reportées dans le plan $\left(\Delta \varepsilon_{\mathrm{a}} \mathrm{SA}, D\right)$ de la Figure $9 \mathrm{~b}$. 
Les points expérimentaux produits par l'essai triaxial cyclique sont groupés autour de la courbe $I_{\mathrm{p}}=15$, pour un indice de plasticité qui n'est pas trop éloigné de celui du limon. Il faut noter l'amortissement minimal $D_{\min }$ non nul, qui est inclus implicitement dans la formulation, ne semble pas reproduit par l'expérience. À l'opposé, l'amortissement prévu par les lois empiriques croît vers une asymptote, alors que la mesure recueillie pendant la séquence de cycles $y_{8}$ se trouve en retrait par rapport à celle de la séquence $y_{7}$ (Tab. 3).

\section{Conclusion}

Une méthode d'identification des paramètres dynamiques d'un sol est proposée. Elle s'applique aux courbes contraintes et déformations qui sont enregistrées au cours d'un essai triaxial cyclique. Des approximations en série de Fourier des courbes expérimentales sont calculées au préalable sur un intervalle donné des enregistrements. Partant de ces courbes et après filtrage pour éliminer le bruit des mesures, il est possible d'identifier les paramètres caractéristiques des boucles d'hystérésis recueillies pendant les séquences de cycles successives appliquées à l'éprouvette. La méthode trouve un intérêt dans sa capacité à décrire des boucles distordues qui s'observent quand l'amplitude cyclique de la déformation imposée augmente et produit une réponse de plus en plus non-linéaire.

Un essai triaxial cyclique réalisé sur un limon prélevé dans son état naturel a servi d'exemple d'illustration de la méthode. Les caractéristiques d'état du limon ont été précisées, puis ses propriétés physiques. Un essai triaxial monotone non drainé a fourni un éclairage préliminaire du comportement mécanique du limon. L'essai triaxial cyclique est présenté ensuite et ses résultats sont commentés. Les propriétés dynamiques du limon sont identifiées pour obtenir la courbe de dégradation du module d'Young avec l'amplitude des déformations et la courbe d'évolution de l'amortissement. Ces propriétés dynamiques ne s'éloignent pas trop des propriétés tirées de formulations empiriques décrites dans la littérature.

La méthode proposée pour identifier les propriétés dynamiques à partir des courbes triaxiales semble donner satisfaction. Elle est adaptable aux différentes modalités pratiques de mise en œuvre de l'essai triaxial (amplitude, fréquences, durée des séquences de cycles). Elle peut s'appliquer à toute ou partie des séquences de cycles. Elle a été mise en œuvre avec succès pour exploiter de nombreux essais triaxiaux cycliques effectués sur des limons et des argiles, voire des sables limoneux, testés dans leur état naturel.

\section{Références}

American Society for Testing and Materials (ASTM). 1996. ASTP standard D3999-11, standard test methods for the determination of the modulus and damping properties of soils using the cyclic triaxial apparatus. In: Annual book of ASTM standards. ASTM International, $14 \mathrm{p}$.

Association française de normalisation (AFNOR). 1994a. Essais à l'appareil triaxial de révolution généralités définitions. Norme française NF P 94-070, octobre 1994, 21 p.

Association française de normalisation (AFNOR). 1994b. Essais à l'appareil triaxial de révolution. Appareillage. Préparation des éprouvettes. Essais UU non consolidé non drainé. Essai $\mathrm{CU}+u$ consolidé non drainé avec mesure de la pression interstitielle. Essai CD consolidé drainé. Norme française NF P 94-074, octobre 1994, 36 p.

Bui MT, Clayton CRI, Priest JA. 2007. Effects of particule shape on $G_{\max }$ of geomaterials. In: Proc. 4th International Conference on Earthquake Geotechnical Engineering (Thessaloniki, Greece), paper No. 1536.

Darendeli MB. 2001. Development of a new family of normalized modulus reduction and material damping curves. $\mathrm{PhD}$ dissertation, University of Texas at Austin (Austin, Texas).

Dyvik R, Madshus C. 1986. Lab measurements of $G_{\max }$ using bender elements. Advances in the art of testing soils under cyclic conditions. In: Proc. ASCE Convention (Detroit, Michigan), pp. 186-196.

El Mohtar CS, Drnevich V, Santagata M, Bobet A. 2013. Combined resonant column and cyclic triaxial tests for measuring undrained shear modulus reduction of sand with plastic fines. Geotech Test $J$ 36: $1-8$.

Guerreiro P, Kontoe S, Taborda D. 2012. Comparative study of stiffness reduction and damping curves. In: Proc. 15th World Conference on Earthquake Engineering (Lisbon, Portugal).

Hardin BO, Drnevich VP. 1972a. Shear modulus and damping in soils: measurement and parameter effects. Terzaghi lecture. J Soil Mech Found Div 98: 603-624.

Hardin BO, Drnevich VP. 1972b. Shear modulus and damping in soil: design equations and curves. J Soil Mech Found Div 98: 667-692.

Ishibashi I, Zhang XJ. 1993. Unified dynamic shear moduli and damping ratios of sand and clay. Soils Found 33: 182 191.

Iwasaki T, Tatsuoka F. 1977. Effects of grain size and grading on dynamic shear moduli of sands. Soils Found 17: 19-35.

Iwasaki T, Tatsuoka F, Takagi Y. 1978. Shear moduli of sands under cyclic torsional shear loading. Soils Found 18: 39-56.

Kokusho T. 1980. Cyclic triaxial test of dynamic soil properties for wide strain range. Soils Found 20: 45-60.

Kokusho T, Yoshida Y, Esashi Y. 1982. Dynamic properties of soft clay for wide strain range. Soils Found 22: 1-18.

Kumar SS, Krishna AM, Dey A. 2015. Cyclic response of sand using stress controlled cyclic triaxial tests. In: Proc. 50th India Geotech. Conf. (Pune, Maharashtra, India).

Liao T, Massoudi N, McHood M, Stokoe KH, Jung MJ, Menq FY. 2013. Normalized shear modulus of compacted gravel. In: Proc. 18th Int. Conf. on Soil Mech. and Geotech. Engrg. (Paris, France).

Matasovic N, Vucetic M. 1992. A pore pressure model for cyclic straining of clay. Soils Found 32: 156-173.

Matasovic N, Vucetic M. 1995. Generalized cyclic-degradation pore-pressure generation model for clays. J Geotech Eng 121: $33-42$.

Nogami Y, Murono Y, Morikawa H. 2012. Nonlinear hysteresis model taking into account S-shaped hysteresis loop and its standard parameters. In: Proc. 15th World Conference on Earthquake Engineering (Lisbon, Portugal).

Rollins KM, Evans M, Diehl N, Daily W. 1998. Shear modulus and damping relationships for gravels. J Geotech Geoenviron Eng 124: 396-405.

Seed HB, Idriss IM. 1970. Soil moduli and damping factors for dynamic response analyses. Report EERC 70-10. Berkeley: Earthquake Engineering Research Centre, University of California.

Seed HB, Wong RT, Idriss IM, Tokimatsu K. 1986. Moduli and damping factors for dynamic analysis of cohesionless soils. $J$ Geotech Eng 112: 1016-1032. 
Shirley DJ, Hampton LD. 1978. Shear-wave measurements in laboratory sediments. J Acoust Soc Am 63: 607-613.

Stokoe KH, Darendeli MB, Andrus RD, Brown LT. 1999. Dynamic soil properties: laboratory, field and correlation studies. In: Proc. 2nd Int. Conf. on Earthquake Geotechnical Engineering (Lisbon, Portugal), Vol. 3, pp. 811-845.

Stokoe KH, Darendeli MB, Gilbert RB, Menq FY, Choi WK. 2004. Development of a new family of normalized modulus reduction and material damping curves. In: NSF/PEER Int. Workshop on Uncertainties in Nonlinear Soil Properties and their Impact on Modeling Dynamic Soil Response, Univ. of California (Berkeley, California).

Sun JI, Goleskorkhi R, Seed HB. 1988. Dynamic moduli and damping ratios for cohesive soils. In: Rep. No. UCB/EERC-88/15, Univ. of California at Berkeley (Berkeley, California).
Vardanega PJ, Bolton MD. 2013. Stiffness of clays and silts: normalizing shear modulus and shear strain. J Geotech Geoenviron Eng 139: 1575-1589.

Vucetic M, Dobry R. 1991. Effect of soil plasticity on cyclic response. $J$ Geotech Eng 117: 89-107.

Vucetic M, Lanzo G, Doroudian M. 1998. Damping at small strains in cyclic simple shear test. J Geotech Geoenviron Eng 124: 585-594.

Zen K, Umehara Y, Hamada K. 1978. Laboratory tests and in situ seismic survey on vibratory shear modulus of clayey soils with various plasticities. In: Proc. 5th Japanese Earthquake Engrg. Symp. (Japan), pp. 721-728.

Zhang J, Andrus RD, Juang CH. 2005. Normalized shear modulus and material damping ratio relationships. J Geotech Geoenviron Eng 131: 453-464.

Citation de l'article : Jean-François Serratrice. Mesures des propriétés cycliques des sols limoneux ou argileux au laboratoire. Rev. Fr. Geotech. 2016, 148, 1. 